\title{
No variation for Wolbachia-induced hybrid breakdown in two populations of a spider mite
}

\author{
F. VALA ${ }^{1,2, *}$, J.A.J. BREEUWER ${ }^{2}$ and M.W. SABELIS ${ }^{2}$ \\ ${ }^{1}$ Department of Biology, University College of London, Wolfson House 4 Stephenson Way, NW1 $2 H E$ \\ London, United Kingdom; ${ }^{2}$ Institute for Biodiversity and Ecosystem Dynamics, University of Amster- \\ dam, P.O. Box 94084, 1090 GB Amsterdam, The Netherlands; *Author for correspondence (e-mail: \\ f.vala@ucl.ac.uk; phone: (44 20) 7679 5095; fax: (44 20) 7383 2048)
}

Received 1 October 2002; accepted in revised form 2 January 2003

Key words: Hybrid breakdown, Reproductive isolation, Spider mites, Wolbachia

\begin{abstract}
Wolbachia are cytoplasmically transmitted bacteria that infect several species of mites. In the two-spotted spider mite Tetranychus urticae Koch this symbiont can induce reproductive incompatibility. Wolbachia-induced reproductive incompatibility is observed in crosses between Wolbachia-infected (W) males and uninfected (U) females. This incompatibility is expressed in F1 broods as male-biased sex ratios, an effect called cytoplasmic incompatibility (CI). However, in the two-spotted spider mite, Wolbachia-induced reproductive incompatibility may extend to the F2: broods of virgin F1 females from $\mathrm{U} \times \mathrm{W}$ crosses sometimes suffer increased mortality rates. This F2 effect is called hybrid breakdown (HB). Several isofemale lines derived from mites collected from rose and cucumber plants had been previously tested for CI. Here we report on the results obtained for HB.
\end{abstract}

\section{Introduction}

Wolbachia are cytoplasmically transmitted bacteria that occur in several arthropod and nematode hosts. These bacteria are obligate endosymbionts, i.e. they cannot live outside the cytoplasm of a host cell. Consequently, Wolbachia colonize new hosts predominantly through transmission from females (the egg producing sex) to their offspring. The reproductive success of Wolbachia is therefore tightly coupled to the reproductive success of the female where it resides. Wolbachia have been called sexual parasites (Werren 1997): they manipulate host reproduction to their benefit - i.e., in such a way that the number of infected females in the host population, directly or indirectly, increases. Depending on the species, and sometimes on the population of a species, these manipulations include feminization of infected males, induction of parthenogenesis in infected females, male-killing and cytoplasmic incompatibility (for a review see Stouthamer et al. (1999)). In the phytophagous haplodiploid two-spotted spider mite Tetranychus urticae Koch, Wolbachia can induce both cytoplasmic incompatibility (Breeuwer 1997; Perrot-Minnot et al. 2002; Vala et al. 2000, 2002) and hybrid breakdown (Vala et al. 2000).

Cytoplasmic incompatibility (CI) is expressed in crosses between Wolbachia infected $(\mathrm{W})$ males and uninfected $(\mathrm{U})$ females (reviewed by Hoffmann and Turelli 
(1997) and Stouthamer et al. (1999)). CI is not induced if the same Wolbachia strain that was present in the male is present in the fertilized egg (i.e. CI is not induced in $+\mathrm{W} \times \mathrm{W}$ o crosses). CI reduces the fitness of uninfected females (which are incompatible with $\mathrm{W}$-males) relative to infected females (which are compatible with both $\mathrm{W}$ - and U-males). As a result, the frequency of infected females increases in the host population.

Cytological studies of CI in Nasonia wasps (Reed and Werren 1995), and Droso-


set of chromosomes does not segregate properly in early mitotic divisions. This results in haploid or aneuploid embryos. In diploid species, haploid and aneuploid embryos abort, thus CI is expressed as increased F1 mortality. In haplodiploids, where females are diploid and males are haploid, haplodized eggs develop as males. Depending on the degree of aneuploidy eggs may (Breeuwer 1997): (1) develop as a male (if eggs revert to the haploid state); (2) die if haplodization is not complete but insufficient to develop as a female; or (3) develop as an aneuploid female (explained below). Mortality of aneuploid embryos may explain why, in most haplodiploid species, CI is expressed as a bias of F1 sex ratio towards males associated with an increase in mortality (cf. Breeuwer (1997) and Vala et al. (2000), Vavre et al. (2000)).

Although detailed cytological analysis is lacking, crossing experiments using the two-spotted spider mite suggest that aneuploid embryos produced in $\$ \mathrm{U} \times 0^{\top} \mathrm{W}$ crosses may also develop as females (Breeuwer 1997; Vala et al. 2000). Aneuploid females, i.e. females whose (diploid) nuclear genome is incomplete, survive as they have the intact maternal set of chromosomes to compensate for the incomplete paternal set. Meiosis in aneuploid females produces aneuploid gametes. Fully haploid eggs develop as males, but haplo-aneuploid eggs abort. Consequently, tests for aneuploidy consist in scoring the F2 mortality of broods of virgin F1 females of

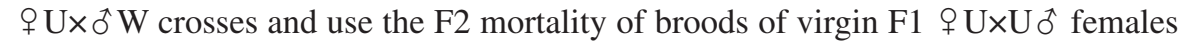
as a control.

Increased mortality among broods of virgin hybrid F1 females is termed hybrid breakdown (HB) in the literature on spider mites (review by Egas et al. (2002)). $\mathrm{HB}$ is typically induced when males and females from two different populations are crossed. However, this phenotype was also observed in virgin F1 daughters from o $\mathrm{U} \times{ }^{\widehat{T}} \mathrm{~W}$ crosses (Vala et al. 2000). In that study parental female and male were from the same population but the mother had been cured of infection by Wolbachia through antibiotic treatment. Like CI, Wolbachia associated HB was not observed when Wolbachia was either present in the parental female and/or absent in the male.

In a recent paper Vala et al. (2002) showed that variability for induction of CI was present in two spider mite populations. In a strain of mites originating from cucumber plants Wolbachia did not induce CI in five isofemale lines tested (Vala et al. (in press)). In this strain infected females tend to produce more female biased sex ratios (Vala et al. (in press)). In the strain of mites collected from rose plants, two types of isofemale lines were isolated. In two lines (R1 and R2) CI was induced, but in another isofemale line (R3) CI was not observed. 
In the present paper we describe experiments that test the effect of Wolbachia on HB for the isofemale lines of both the cucumber and rose strain. The results support the conclusions that (1) Wolbachia has no effect in reproductive compatibility in the cucumber strain; and (2) HB is not observed in line R3, supporting the previous conclusion that in the rose strain there is variation for the effect of Wolbachia on reproductive compatibility. In this strain two types of host-Wolbachia association seem to co-occur: hosts where infection results in reproductive incompatibility of infected males with uninfected females and hosts where it does not.

\section{Material and methods}

\section{Base population}

Two populations of $T$. urticae spider mites were established in the lab. One from mites collected from rose plants (the $\mathrm{R}$ strain, hereafter) in a greenhouse at Aalsmeer, The Netherlands; and another from mites collected from cucumber plants (the C strain, hereafter) obtained from the Institute for Horticultural Plant Breeding in Wageningen, The Netherlands. Since collection, spider mites have been reared on detached leaves of Phaseolus vulgaris (variety 'Arena'). Cultures were maintained, and experiments were performed, in one climate room at $23{ }^{\circ} \mathrm{C}, 60-80 \%$ relative humidity, and 16L:8D photoperiod. A polymerase chain reaction (PCR) assay with Wolbachia-specific primers detected that both strains were infected with Wolbachia (Breeuwer and Jacobs 1996). DNA isolation and PCR were as in Breeuwer (1997). The R- and C-strain are the same as those described in Stouthamer et al. (1999).

\section{Isofemale lines}

Isofemale lines of the two strains were created by taking virgin females from the infected base populations and performing mother to son mating for 4 consecutive generations - for arrenotokous haplodiploid organisms this gives an expected inbreeding coefficient of 0.98 (Hartl 1980). From each inbred isofemale line an uninfected line was created either by tetracycline curing or by heat treatment (Breeuwer (1997) and Van Opijnen and Breeuwer (1999) for further details see Vala et al. (2002)). Isofemale lines cured by tetracycline are labeled 'TET', isofemale lines cured by heat treatment are labeled 'HT'. For one isofemale line (R3) two uninfected sub-lines were established one by curing with tetracycline the other by curing with heat. The 'TET' and 'HT' R3 sub-lines were compared to control for treatment method effects. Previous results have shown that with respect to the F1 generation treatments yield no treatment-specific side effects (Vala et al. 2002). Here we report on F2 results.

Because lines were inbred prior to curing, and thus are expected to be nearly homozygous, differences between the infected and uninfected sub-line of each isofemale line are most likely due to presence of Wolbachia. Sequencing of two 
Wolbachia genes ( $w s p$ and $f t s Z$ ) revealed that sequences were identical in both population (Vala et al. 2002). Thus, the hypothesis that differences between isofemale lines could be due to genetic differences at the nuclear level.

\section{Test for hybrid breakdown}

To detect variation in Wolbachia induced reproductive incompatibility, several isofemale lines from each population were tested for CI (Vala et al. 2002) and HB. Here we report results for the latter. To test for HB we used virgin daughters from all possible crosses between infected and uninfected individuals (i.e. daughters from, $+\times \widehat{0}, \mathrm{~W} \times \mathrm{W}, \mathrm{W} \times \mathrm{U}, \mathrm{U} \times \mathrm{U}$ and $\mathrm{UxW}$ ). The test for $\mathrm{HB}$ consists in scoring the mortality of $\mathrm{F} 2$ broods from virgin $\mathrm{F} 1$ daughters of $q \mathrm{U} \times \sigma^{\top} \mathrm{W}$ parents and compare with the mortality of $\mathrm{F} 2$ broods from virgin daughters of $q \mathrm{U} \times \widehat{\widehat{ }} \mathrm{U}$ parents. If $\mathrm{F} 1$ ( $q \mathrm{U} \times \widehat{\jmath}_{\mathrm{W}}$ ) females are aneuploid, then higher mortality will be observed among their haploid broods. Higher haploid mortality (HB) should not be observed in

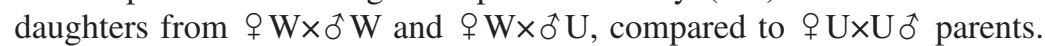

\section{Procedures for all experiments}

Twenty-five to 30 females of each line laid eggs on detached bean leaves (Phaseolus vulgaris) placed on water-soaked cotton wool balls. These females were transferred at three-day intervals to produce age cohorts. Offspring from these cohorts were used in the experiments. All experiments were performed on bean leaf discs ( $1.5 \mathrm{~cm}$ in diameter). Leaf discs were placed on water-soaked cotton wool 'sheets' stretched upon sponges. Sponges were placed on plastic trays and water was added regularly to prevent the leaf discs from drying. In all experiments, crosses and spider mite lines were randomized across sponges.

F1 females were collected, from all crosses, 10-12 d after oviposition. We only used F1 females from parental females that survived the entire period of the preceding CI experiments (also published recently, see Vala et al. (2002)). Females at the last molting stage were collected and transferred individually to leaf discs. After five days, they were transferred to a fresh leaf disc for another $3 \mathrm{~d}$. Offspring (number of F2 males, unhatched eggs and dead individuals) were counted 10-12 d later and used to compute clutch size (CS = number unhatched eggs + number dead + number F2 males), and F2 mortality (mortality = (number unhatched eggs + number of dead) / CS).

\section{Statistical analysis}

Effect of factors was analyzed by MANOVAs on derived variables (i.e. variables computed from what was actually measured in the experiments: clutch size and F2 mortality) because these variables will generally not be independent. We report the MANOVA Wilk's $\lambda$ test statistic. The normality of data was tested graphically and significance was examined using the Shapiro-Wilk test. Homocedasticity (equality of group variances) was tested using Levine's test. In MANOVAs equality of co- 
variance matrices was tested using the Box's test. Non-parametric tests (KruskalWallis) were used when assumptions normality and homocedasticity were violated (provided they could not be solved by transformation). Statistic analysis was performed using SPSS. MANOVAs were followed by a series of univariate ANOVAs. The significance level $\alpha$ of these ANOVAs, $\mathrm{P}=0.05$, was adjusted following the Bonferroni procedure to correct for multiple analysis (Field 2000). Pairwise comparisons were performed using Tukey post-hoc tests.

\section{Results}

\section{Spider mite lines}

Lines established from PCR-negative females in an assay with Wolbachia specific primers, remained negative without further treatment, when tested before and after the experiments. Three rose (R1-3) and five cucumber isofemale lines (C1-5) were used.

\section{Test for hybrid breakdown}

For isofemale line R3, two cured sub-lines were established by curing with tetracycline and by curing with heat treatment. Results with F1 virgin R3-females show no effect of treatment on mortality (Table 1). However, clutch size (and consequently, number of F2 males) was lower for daughters of crosses between 'TET' females and males (Table 1). This effect was only observed in the F2, disappeared if an infected grandfather had been used, and did not seem to be general - i.e. crosses between TET-mites did not consistently produce smaller clutch sizes than crosses between HT-mites in other isofemale lines (cf. Tables 1, 2 and 3). Thus, we are uncertain that the origin of this effect really is the method of curing. The qualitative result with this line did not change with treatment - i.e. neither CI nor HB were induced in crosses between infected males and either type (TET/HT) of female (cf. Vala et al. (2002) for CI; Tables 1 and 2 for HB). Therefore, we conclude that method of curing had no effect other than removal of the symbiont.

Hybrid breakdown, i.e. a significant increase in mortality of F2 males, was not observed in any of the three $\mathrm{R}$ isofemale lines tested (Table 1). However, average mortality increased and the numbers of F2 males decreased in line R1. In this line we did not properly test induction of HB because very few F1 ( $q \mathrm{U} \times 0^{\top} \mathrm{W}$ ) females 'survived' CI - previous results showed that CI is very strong in this line (Vala et al. 2002). So perhaps the statistical test could not detect a significant difference because the sample size of this group of females was so small.

To test whether infected R3 females retained the property of rescuing modified sperm, despite the fact that sperm from infected R3 males is not modified, R3 and R1 mites were crossed. F2 results are presented in Table 2. First, as for other experiments (Table 1), W-R3 males did not induce HB in U-R3 females. Second, 
Table 1. Test for induction of hybrid breakdown: crosses within isofemale lines from the Rose population.

Legend: W, Wolbachia infected; U, uninfected (cured); N, sample sizes; HT, mites cured with heat treatment; TET, mites cured with tertracycline. Variables significant (after Bonferroni correction) according to univariate ANOVAs are marked with an asterisk; identical superscripts $(\mathrm{a}, \mathrm{b}$ or $\mathrm{c}$ ) within columns indicate non-significant differences between crosses at the 5\% level (Tukey test).

\begin{tabular}{|c|c|c|c|c|c|c|c|c|c|c|}
\hline \multirow{3}{*}{$\begin{array}{l}\text { Rose } 1(\mathrm{HT}) \\
\text { F1 female's parents: } \\
(+\times \widehat{ } \times)\end{array}$} & \multirow{3}{*}{$\mathrm{N}$} & \multirow{2}{*}{\multicolumn{3}{|c|}{ Clutch Size* }} & \multirow{2}{*}{\multicolumn{3}{|c|}{ Mortality }} & \multirow{2}{*}{\multicolumn{3}{|c|}{ Number of sons }} \\
\hline & & & & & & & & & & \\
\hline & & mean & \pm & $\mathrm{SE}$ & Mean & \pm & $\mathrm{SE}$ & mean & \pm & SE \\
\hline$(\mathrm{U} \times \mathrm{U})$ & 17 & $26.94^{\mathrm{a}}$ & \pm & 1.88 & 0.33 & \pm & 0.05 & 18.59 & \pm & 1.30 \\
\hline$(\mathrm{U} \times \mathrm{W})$ & 4 & $18.75^{\mathrm{a}}$ & \pm & 7.36 & 0.49 & \pm & 0.16 & 10.75 & \pm & 1.51 \\
\hline$(\mathrm{W} \times \mathrm{W})$ & 13 & $37.15^{\mathrm{b}}$ & \pm & 2.00 & 0.24 & \pm & 0.07 & 28.69 & \pm & 1.71 \\
\hline$(\mathrm{W} \times \mathrm{U})$ & 24 & $34.52^{\mathrm{b}}$ & \pm & 2.81 & 0.29 & \pm & 0.05 & 25.17 & \pm & 1.48 \\
\hline Manova: & \multicolumn{10}{|c|}{$\mathrm{F}_{9,116}=1.99$, Wilk's $\lambda=0.636, \mathrm{P}=0.02$} \\
\hline Anovas: & \multicolumn{4}{|c|}{$\mathrm{F}_{3,53}=7.12, \mathrm{P}<0.001$} & \multicolumn{3}{|c|}{$\mathrm{F}_{3,53}=1.05, \mathrm{~ns}$} & \multicolumn{3}{|c|}{$\mathrm{F}_{3,126}=3.46, \mathrm{~ns}$} \\
\hline \multicolumn{11}{|l|}{ Rose 2 (TET) } \\
\hline F1 female's parents: & $\mathrm{N}$ & \multicolumn{3}{|c|}{ Clutch Size } & \multicolumn{3}{|c|}{ Mortality } & \multicolumn{3}{|c|}{ Number of sons } \\
\hline$(q \times 0)$ & & mean & \pm & SE & mean & \pm & SE & mean & \pm & SE \\
\hline$(\mathrm{U} \times \mathrm{U})$ & 22 & 37.50 & \pm & 2.32 & 0.10 & \pm & 0.04 & 33.41 & \pm & 2.47 \\
\hline$(\mathrm{U} \times \mathrm{W})$ & 16 & 29.94 & \pm & 3.93 & 0.08 & \pm & 0.02 & 27.75 & \pm & 3.75 \\
\hline$(\mathrm{W} \times \mathrm{W})$ & 27 & 36.07 & \pm & 1.38 & 0.19 & \pm & 0.04 & 29.63 & \pm & 1.90 \\
\hline$(\mathrm{W} \times \mathrm{U})$ & 28 & 29.94 & \pm & 2.65 & 0.14 & \pm & 0.03 & 25.42 & \pm & 2.50 \\
\hline Manova: & \multicolumn{10}{|c|}{$\mathrm{F}_{9,212}=1.99$, Wilk's $\lambda=0.82, \mathrm{P}=0.042$} \\
\hline Anovas: & \multicolumn{4}{|c|}{$\mathrm{F}_{3,92}=1.74, \mathrm{~ns}$} & \multicolumn{3}{|c|}{$\mathrm{F}_{3,92}=3.70, \mathrm{~ns}$} & \multicolumn{3}{|c|}{$\mathrm{F}_{3,92}=4.13, \mathrm{~ns}$} \\
\hline \multicolumn{11}{|l|}{ Rose 3 (HT and TET) } \\
\hline F1 female's parents: & $\mathrm{N}$ & \multicolumn{3}{|c|}{ Clutch Size* } & \multicolumn{3}{|c|}{ Mortality } & \multicolumn{3}{|c|}{ Number of sons* } \\
\hline$(q \times 0)$ & & mean & \pm & SE & Mean & \pm & SE & mean & \pm & SE \\
\hline$\left(\mathrm{U}_{\mathrm{HT}} \times \mathrm{U}_{\mathrm{HT}}\right)$ & 21 & $40.90^{\mathrm{b}}$ & \pm & 2.09 & 0.29 & \pm & 0.06 & $30.29^{b}$ & \pm & 3.10 \\
\hline$\left(\mathrm{U}_{\mathrm{HT}} \times \mathrm{W}\right)$ & 14 & $35.31^{\mathrm{b}}$ & \pm & 4.50 & 0.22 & \pm & 0.06 & $29.12^{\mathrm{b}}$ & \pm & 4.15 \\
\hline$(\mathrm{W} \times \mathrm{W})$ & 11 & $33.55^{\mathrm{a}, \mathrm{b}}$ & \pm & 4.25 & 0.32 & \pm & 0.07 & $21.73^{\mathrm{a}, \mathrm{b}}$ & \pm & 3.23 \\
\hline$\left(\mathrm{W} \times \mathrm{U}_{\mathrm{HT}}\right)$ & 20 & $38.50^{\mathrm{b}}$ & \pm & 2.09 & 0.25 & \pm & 0.05 & $29.90^{\mathrm{b}}$ & \pm & 2.71 \\
\hline$\left(\mathrm{U}_{\mathrm{TET}} \times \mathrm{U}_{\mathrm{TET}}\right)$ & 23 & $26.50^{\mathrm{a}}$ & \pm & 2.87 & 0.41 & \pm & 0.06 & $16.54^{\mathrm{a}}$ & \pm & 2.64 \\
\hline$\left(\mathrm{U}_{\mathrm{TET}} \times \mathrm{W}\right)$ & 15 & $28.60^{\mathrm{a}, \mathrm{b}}$ & \pm & 4.05 & 0.32 & \pm & 0.07 & $20.67^{\mathrm{a}, \mathrm{b}}$ & \pm & 3.24 \\
\hline$(\mathrm{W} \times \mathrm{W})$ & 27 & $32.41^{\mathrm{a}, \mathrm{b}}$ & \pm & 2.12 & 0.26 & \pm & 0.05 & $24.59^{\mathrm{a}, \mathrm{b}}$ & \pm & 2.21 \\
\hline$\left(\mathrm{W} \times \mathrm{U}_{\mathrm{TET}}\right)$ & 26 & $30.22^{\mathrm{a}, \mathrm{b}}$ & \pm & 2.18 & 0.26 & \pm & 0.04 & $23.63^{\mathrm{a}, \mathrm{b}}$ & \pm & 2.17 \\
\hline Manova: & \multicolumn{10}{|c|}{$\mathrm{F}_{21,417}=3.96$, Wilk's $\lambda=0.77, \mathrm{P}=0.008$} \\
\hline Anovas: & \multicolumn{4}{|c|}{$\mathrm{F}_{7,154}=3.82, \mathrm{P}=0.001$} & \multicolumn{3}{|c|}{$\mathrm{F}_{7,154}=1.01, \mathrm{~ns}$} & \multicolumn{3}{|c|}{$\mathrm{F}_{7,154}=3.62, \mathrm{P}=0.001$} \\
\hline
\end{tabular}

W-R1 males mated to U-R1 females did not induce a statistically significant increase in F2 mortality, even though the average mortality of these crosses was almost double that of the control - here again, because few females 'survive' CI, the lack of statistical significance may be due to the small number of F1 ( $q \mathrm{U} \times{ }^{\top} \mathrm{W}$ ) females tested. Third, some degree of nuclear incompatibility seems to exist be- 
Table 2. Hybrid breakdown test for crosses between R1 and R3 isofemale lines (for table legend, see Table 1).

\begin{tabular}{|c|c|c|c|c|c|c|c|}
\hline \multirow{2}{*}{ 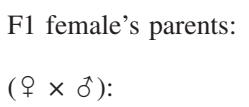 } & \multirow[t]{2}{*}{$\mathrm{N}$} & \multicolumn{3}{|c|}{ Mortality* } & \multicolumn{3}{|c|}{ Number of sons* } \\
\hline & & mean & \pm & SE & mean & \pm & SE \\
\hline \multicolumn{8}{|c|}{ Does $R 1 W$ induce $H B$ in $R 1 U$ ? No. } \\
\hline $\mathrm{R} 1 \mathrm{U} \times \mathrm{R} 1 \mathrm{U}$ & 46 & $0.21^{\mathrm{a}, \mathrm{b}}$ & \pm & 0.04 & $28.16^{\mathrm{a}, \mathrm{c}, \mathrm{d}}$ & \pm & 1.84 \\
\hline $\mathrm{R} 1 \mathrm{U} \times \mathrm{R} 1 \mathrm{~W}$ & 15 & $0.39^{\mathrm{b}, \mathrm{c}}$ & \pm & 0.06 & $21.67^{\mathrm{a}, \mathrm{b}, \mathrm{c}}$ & \pm & 3.00 \\
\hline \multicolumn{8}{|c|}{ Does $R 1 W$ induce $H B$ in $R 3 U$ ? No. } \\
\hline $\mathrm{R} 3 \mathrm{U} \times \mathrm{R} 1 \mathrm{U}$ & 31 & $0.63^{\mathrm{a}, \mathrm{c}}$ & \pm & 0.03 & $12.58^{\mathrm{a}, \mathrm{b}}$ & \pm & 1.45 \\
\hline $\mathrm{R} 3 \mathrm{U} \times \mathrm{R} 1 \mathrm{~W}$ & 16 & $0.52^{\mathrm{c}, \mathrm{d}}$ & \pm & 0.09 & $11.67^{\mathrm{a}, \mathrm{b}}$ & \pm & 0.03 \\
\hline \multicolumn{8}{|c|}{ (Can $\mathrm{R} 3 \mathrm{~W}$ rescue R1W? Yes) } \\
\hline$(\mathrm{R} 3 \mathrm{~W} \times \mathrm{R} 1 \mathrm{~W}$ & 40 & $0.36^{\mathrm{b}, \mathrm{c}}$ & \pm & 0.04 & $21.30^{\mathrm{a}, \mathrm{b}, \mathrm{c}}$ & \pm & 2.00 \\
\hline \multicolumn{8}{|c|}{ Does $\mathrm{R} 3 \mathrm{~W}$ induce $\mathrm{HB}$ in $\mathrm{R} 3 \mathrm{U}$ ? No. } \\
\hline $\mathrm{R} 3 \mathrm{U} \times \mathrm{R} 3 \mathrm{U}$ & 26 & $0.17^{\mathrm{a}}$ & \pm & 0.04 & $34.08^{\mathrm{d}}$ & \pm & 3.43 \\
\hline $\mathrm{R} 3 \mathrm{U} \times \mathrm{R} 3 \mathrm{~W}$ & 22 & $0.29^{\mathrm{a}, \mathrm{b}}$ & \pm & 0.05 & $24.65^{\mathrm{c}, \mathrm{d}}$ & \pm & 2.92 \\
\hline \multicolumn{8}{|c|}{ Does R3W induce HB in R1U? No. } \\
\hline $\mathrm{R} 1 \mathrm{U} \times \mathrm{R} 3 \mathrm{U}$ & 37 & $0.60^{\mathrm{c}, \mathrm{d}}$ & \pm & 0.03 & $12.68^{\mathrm{a}, \mathrm{b}}$ & \pm & 1.14 \\
\hline $\mathrm{R} 1 \mathrm{U} \times \mathrm{R} 3 \mathrm{~W}$ & 28 & $0.68^{\mathrm{c}}$ & \pm & 0.03 & $9.68^{\mathrm{a}}$ & \pm & 1.29 \\
\hline Manova: & \multicolumn{7}{|c|}{$\mathrm{F}_{16,478}=10.00$, Wilk's $\lambda=0.56, \mathrm{P}<0.001$} \\
\hline Anovas: & \multicolumn{4}{|c|}{$\mathrm{F}_{8,248}=15.24, \mathrm{P}<0.001$} & \multicolumn{3}{|c|}{$\mathrm{F}_{8,248}=19.51, \mathrm{P}<0.001$} \\
\hline
\end{tabular}

tween the two lines: daughters of (reciprocal) between-line crosses had higher mortality among their haploid broods than daughters of within-line crosses.

In summary, the results support the conclusion drawn previously that Wolbachia in R3 females effectively rescues CI induced by infected R1 males, so that no HB is observed, and that variation exists in this strain for the effects associated with infection by Wolbachia in males.

Although in isofemale line C5 higher mean F2 mortality was observed (Table 3), this effect was not statistically significant. Thus, the cucumber isofemale lines tested did not show HB associated with presence of Wolbachia in males. (The small sample size in some crosses of lines C5 and C2 are due to the unexpected early emergence of $\mathrm{F} 1$ females in these crosses - it is possible, although we have not tested it, that Wolbachia infected females develop faster). In C-lines, like in the C base population (Vala et al. (in press)) Wolbachia is not associated with induction of reproductive incompatibility.

Finally, the possibility exists that our results could be confounded due to high (or sex dependent) mortality introduced by inbreeding, as is observed in the spider mite Schizotetranychus miscanthi (Saito et al. 2000). In a separate paper (Vala et al. (in press)) we studied the effects of inbreeding on several life-history traits. Very briefly, the results in review show an effect of inbreeding on female longevity and 
Table 3. Test for induction of hybrid breakdown: crosses within isofemale lines from the Cucumber population (for table legend, see Table 1).

\begin{tabular}{|c|c|c|c|c|c|c|c|c|c|c|}
\hline \multirow{3}{*}{$\begin{array}{l}\text { Cucumber } 1 \text { (TET) } \\
\text { F1 female's parents: } \\
(q \times \widehat{o})\end{array}$} & \multirow{3}{*}{$\mathrm{N}$} & \multirow{2}{*}{\multicolumn{3}{|c|}{ Clutch Size }} & \multirow{2}{*}{\multicolumn{3}{|c|}{ Mortality }} & \multirow{2}{*}{\multicolumn{3}{|c|}{ Number of sons }} \\
\hline & & & & & & & & & & \\
\hline & & mean & \pm & SE & Mean & \pm & SE & mean & \pm & SE \\
\hline$(\mathrm{U} \times \mathrm{U})$ & 26 & 33.78 & \pm & 2.25 & 0.39 & \pm & 0.05 & 21.41 & \pm & 2.35 \\
\hline$(\mathrm{U} \times \mathrm{W})$ & 30 & 35.42 & \pm & 2.51 & 0.34 & \pm & 0.04 & 24.90 & \pm & 2.38 \\
\hline$(\mathrm{W} \times \mathrm{W})$ & 28 & 31.90 & \pm & 3.08 & 0.30 & \pm & 0.06 & 24.70 & \pm & 3.04 \\
\hline$(\mathrm{W} \times \mathrm{U})$ & 24 & 37.33 & \pm & 2.05 & 0.22 & \pm & 0.03 & 29.38 & \pm & 2.22 \\
\hline Manova: & \multicolumn{10}{|c|}{$\mathrm{F}_{9,248}=1.12$, Wilk's $\lambda=0.91, \mathrm{~ns}$} \\
\hline \multicolumn{11}{|l|}{ Cucumber 2 (TET) } \\
\hline \multirow{2}{*}{$\begin{array}{l}\text { F1 female's parents: } \\
\left(\uparrow \times 0^{\hat{\top}}\right)\end{array}$} & \multirow[t]{2}{*}{$\mathrm{N}$} & \multicolumn{3}{|c|}{ Clutch Size } & \multicolumn{3}{|c|}{ Mortality } & \multicolumn{3}{|c|}{ Number of sons } \\
\hline & & mean & \pm & SE & mean & \pm & SE & mean & \pm & SE \\
\hline$(\mathrm{U} \times \mathrm{U})$ & 8 & 30.63 & \pm & 5.21 & 0.44 & \pm & 0.11 & 20.13 & \pm & 5.33 \\
\hline$(\mathrm{U} \times \mathrm{W})$ & 9 & 28.00 & \pm & 3.80 & 0.31 & \pm & 0.06 & 18.50 & \pm & 2.25 \\
\hline$(\mathrm{W} \times \mathrm{W})$ & 24 & 33.54 & \pm & 1.27 & 0.32 & \pm & 0.05 & 22.83 & \pm & 2.02 \\
\hline$(\mathrm{W} \times \mathrm{U})$ & 18 & 31.44 & \pm & 1.43 & 0.29 & \pm & 0.04 & 22.56 & \pm & 1.54 \\
\hline Manova: & \multicolumn{10}{|c|}{$\mathrm{F}_{9,129}=1.05$, Wilk's $\lambda=0.41, \mathrm{~ns}$} \\
\hline \multicolumn{11}{|l|}{ Cucumber 3 (HT) } \\
\hline F1 female's parents: & $\mathrm{N}$ & \multicolumn{3}{|c|}{ Clutch Size (CS) } & \multicolumn{3}{|c|}{ Mortality (M) } & \multicolumn{3}{|c|}{ Number of sons (Ns) } \\
\hline$\left(q \times 0^{\top}\right)$ & & mean & \pm & SE & Mean & \pm & SE & mean & \pm & SE \\
\hline$(\mathrm{U} \times \mathrm{U})$ & 24 & 36.71 & \pm & 2.77 & 0.27 & \pm & 0.07 & 30.71 & \pm & 3.28 \\
\hline$(\mathrm{U} \times \mathrm{W})$ & 16 & 34.00 & \pm & 3.49 & 0.14 & \pm & 0.04 & 29.81 & \pm & 3.43 \\
\hline$(\mathrm{W} \times \mathrm{W})$ & 30 & 39.74 & \pm & 2.16 & 0.15 & \pm & 0.03 & 33.90 & \pm & 2.38 \\
\hline$(\mathrm{W} \times \mathrm{U})$ & 24 & 37.20 & \pm & 2.26 & 0.13 & \pm & 0.03 & 32.40 & \pm & 2.37 \\
\hline Kruskal-Wallis & & $\mathrm{CS}, \chi$ & -1 & $4, \mathrm{~ns}$ & $\mathrm{M}, \chi$ & & $9, \mathrm{~ns}$ & Ns, & - & $1, \mathrm{~ns}$ \\
\hline \multicolumn{11}{|l|}{ Cucumber 4 (TET) } \\
\hline \multirow{2}{*}{$\begin{array}{l}\text { F1 female's parents: } \\
\left(\$ \times 0^{\star}\right)\end{array}$} & \multirow[t]{2}{*}{$\mathrm{N}$} & \multicolumn{3}{|c|}{ Clutch Size (CS) } & \multicolumn{3}{|c|}{ Mortality (M) } & \multicolumn{3}{|c|}{ Number of sons (Ns) } \\
\hline & & mean & \pm & SE & mean & \pm & SE & mean & \pm & SE \\
\hline$(\mathrm{U} \times \mathrm{U})$ & 19 & 42.79 & \pm & 1.74 & 0.26 & \pm & 0.05 & 30.95 & \pm & 1.90 \\
\hline$(\mathrm{U} \times \mathrm{W})$ & 18 & 39.61 & \pm & 2.09 & 0.28 & \pm & 0.04 & 28.50 & \pm & 2.25 \\
\hline$(\mathrm{W} \times \mathrm{W})$ & 30 & 39.90 & \pm & 1.98 & 0.25 & \pm & 0.03 & 30.03 & \pm & 2.15 \\
\hline$(\mathrm{W} \times \mathrm{U})$ & 41 & 37.59 & \pm & 1.97 & 0.26 & \pm & 0.03 & 27.59 & \pm & 1.64 \\
\hline Kruskal-Wallis & & $\mathrm{CS}, \chi$ & ${ }_{3}=2$ & $6, \mathrm{~ns}$ & $\mathrm{M}, \chi$ & $=0$. & $6, \mathrm{~ns}$ & Ns, & 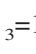 & ns \\
\hline \multicolumn{11}{|l|}{ Cucumber 5 (TET) } \\
\hline F1 female's parents: & $\mathrm{N}$ & Clutc & $\mathrm{Siz}$ & (CS) & & lity & & Num & of & $\mathrm{s}(\mathrm{Ns})$ \\
\hline$\left(\uparrow \times 0^{\hat{*}}\right)$ & & mean & \pm & SE & mean & \pm & SE & mean & \pm & SE \\
\hline$(\mathrm{U} \times \mathrm{U})$ & 17 & 26.94 & \pm & 1.88 & 0.34 & \pm & 0.05 & 18.59 & \pm & 2.17 \\
\hline$(\mathrm{U} \times \mathrm{W})$ & 4 & 18.75 & \pm & 7.36 & 0.49 & \pm & 0.16 & 10.75 & \pm & 5.62 \\
\hline$(\mathrm{W} \times \mathrm{W})$ & 13 & 37.15 & \pm & 2.00 & 0.24 & \pm & 0.07 & 28.69 & \pm & 3.33 \\
\hline$(\mathrm{W} \times \mathrm{U})$ & 21 & 34.52 & \pm & 2.81 & 0.29 & \pm & 0.05 & 25.17 & \pm & 3.00 \\
\hline Manova: & & & & $\mathrm{F}_{9,151}$ & $=0.514$, & ilk' & $\lambda=0$. & $\mathrm{ns}$ & & \\
\hline
\end{tabular}


female fecundity (F1 clutch size) whereas variation in F1 mortality and F1 sex ratio is mostly explained by isofemale line (i.e. genotype). In this paper we study mortality and fecundity of virgin females. The difference is important because effects of inbreeding could be stronger on all-haploid broods due to expression of deleterious recessive alleles in haploid genomes. Thus we draw a rough comparison of the present results with data published previously for the outbred $\mathrm{R}$ base population (see Vala et al. 2000), and the outbred C population (see Vala et al. (in press)). This suggests that females from inbred lines produce smaller clutches, whereas effects on mortality seem better explained by isofemale line (i.e. genotype) than by inbreeding. This is perfectly in line with the conclusions drawn from results obtained with the F1 (Vala et al. (in press)). We therefore conclude that inbreeding is unlikely to play a role in the results reported here.

\section{Discussion}

The results reported here confirm previous results obtained with the cucumber and rose strain of the two-spotted spider mite Tetranychus urticae Koch, regarding variation for the effect of infection by Wolbachia between and within these populations (cf. Vala et al. 2000, 2002). Variation for the effects of Wolbachia in T. urticae has been independently documented by another research group (cf. PerrotMinnot et al. (2002)).

Crosses within the R-strain confirm that this strain is polymorphic for the effect of infection by Wolbachia bacteria, i.e. two phenotypes associated with the infection have been isolated (Vala et al. 2002). Previous experiments showed that CI was not present in crosses between uninfected R3 females and infected R3 males, whereas CI was induced in crosses between uninfected females and infected males of isofemale lines R1 and R2 (Vala et al. 2002). Further crossing experiments demonstrated that presence of Wolbachia-host in R3 females rescued CI induced by infected-R1 males (Vala et al. 2002). In line with those results, we show here that $\mathrm{HB}$ is not induced in crosses between uninfected R3 females and infected R3 males (Table 1), showing that indeed Wolbachia has no effect on reproductive incompatibility in this line. Moreover, HB was also not observed in crosses between uninfected R3 females and infected R1 males (Table 2), thus the rescuing effect from CI seems complete.

The absence of reproductive incompatibility between R3-infected males and uninfected females is unlikely to be due to decreased symbiont densities in this line, compared to R1 and R2. If sperm modification by Wolbachia, to induce CI, depends on the density of the bacteria in males then, most likely, rescue of CI (i.e. of chromosomes from infected males) by Wolbachia in eggs will also be density dependent. Thus if Wolbachia induced reproductive incompatibility is not observed in $\mathrm{R} 3$ due to low densities of the symbiont in this line but is observed in R1 and R2 due to higher densities of the symbiont in those lines, then incompatibility of infected R3 females with infected R1 males should follow - which was not the case. 
Thus we think it is unlikely that observed polymorphism of Wolbachia induced incompatibility within R-lines could be explained by differences in symbiont density. Rather we favor the possibility that the ability to modify sperm has been lost in this line either because the host resists modification or because the symbiont has lost the ability to produce it. The genetic basis of this effect, like the genetic basis of CI, remains to be established.

In summary, in the rose strain host-Wolbachia associations that result in reproductive incompatibility between infected males and uninfected females may co-occur with associations where incompatibility is not observed.

A curious result with the R-strain is the appearance of reproductive isolation i.e. nuclear HB (De Boer 1985) - between R1 and R3 compared to crosses within the lines. Reproductive isolation between spider mite populations is commonly reported and sometimes, but clearly not always, it correlates with geographic distance or host-plant (De Boer 1985). Fast evolution of spider mite populations (e.g. spread of a pesticide resistance trait) is probably accounted for by the haplodiploid mode of reproduction of this species (Crozier 1985). But the molecular mechanism underlying rapid appearance of incompatibility types remains unknown. It is possible that new compatibility types arise frequently in spider-mite populations but are rapidly selected against if reproductive isolation does not confer a selective advantage to individuals possessing it (i.e. if production of hybrids is advantageous). Once isolated and inbred R1 and R3 lines were not in contact with each other and each line was strongly inbred, thus these 'incompatibility traits' may have become fixed. [The puzzling observation unaccounted for by this hypothesis is that presence of Wolbachia in the R3 female was associated with reduced between-line nuclear incompatibility HB effect-however this effect was not statistically significant even though sample size was sufficiently large (cf. row 3, 4 and 5 of Table 2) and should probably be ignored.]

Crosses within isofemale lines derived from the cucumber strain revealed that presence of Wolbachia in males did not result in HB in any isofemale line tested (Table 3). The same result is observed in crosses within the cucumber base population (Vala et al. (in press)). How does an infection that does not induce reproductive incompatibility with uninfected females maintain itself in the host population? Previous results showed that Wolbachia induces a sex ratio bias towards females (Stouthamer et al. 1999; Vala et al. 2002). This effect allows Wolbachia to spread in a haplodiploid host population (Egas et al. 2002).

The between-line and between-population variation reported here is likely to reflect genetic differences because environmental conditions were constant throughout experiments. It is presently unclear if the variation found for the effect of Wolbachia on reproductive isolation is due to the host, the symbiont or both. Sequences of two Wolbachia genes (wsp and $f s t Z$ ) are identical in the $\mathrm{C}$ and $\mathrm{R}$ populations of this host species (Vala et al. 2002). Similarly, Perrot-Minnot et al. (2002) report identical wsp sequences in Wolbachia from three infected populations of T. urticae spider mites that show different degrees of CI. Thus, there is the strong possibility that the phenotypic discrepancy for the effects of the infection found in this hostspecies is due to genotypic differences at the host level. 
The origin of variation in Wolbachia induced incompatibility could be examined by performing introgression experiments: placing the cytoplasm of R3 in the nuclear background of R1 and R2, and vice-versa, followed by the CI and HB diagnose crosses would establish if the effect is host or symbiont derived (or both). A similar experiment, but through microinjection, could be performed to test the effect of the $\mathrm{R}$ and $\mathrm{C}$ nuclear and cytoplasmic backgrounds (introgression through crossing is not likely to produce results due to the existing nuclear incompatibility between these populations-see Vala et al. (2000)). Given the variation observed, the Wolbachia association with the two-spotted spider mite remains one model system to study the evolution of endosymbiosis.

\section{Acknowledgements}

F. Vala was supported by Fundação para a Ciencia e Tecnologia (scholarship reference: Praxis XXI/BD/9678/96). We acknowledge two anonymous referees for valuable comments on a previous version of this paper.

\section{References}

Breeuwer J.A.J. 1997. Wolbachia and cytoplasmic incompatibility in the spider mite Tetranychus urticae and T turkestani. Heredity 79: 41-47.

Breeuwer J.A.J. and Jacobs G. 1996. Wolbachia: intracellular manipulators of mite reproduction. Exp. Appl. Acarol. 20: 421-434.

Callaini G., Dallai R. and Riparbelli M.G. 1997. Wolbachia-induced delay of paternal chromatin condensation does not prevent maternal chromosomes from entering anaphase in incompatible crosses of Drosophila simulans. Journal of Cell Science 110: 271-280.

Crozier R.H. 1985. Adaptive consequences of male-haploidy. In: Helle W. and Sabelis M.W. (eds), Spider mites: their biology, natural enemies and control. Elsevier, Amsterdam.

De Boer R. 1985. Reproductive Barriers. In: Helle W. and Sabelis M.W. (eds), Spider mites: their biology, natural enemies and control. Elsevier, Amsterdam.

Egas M., Vala F. and Breeuwer J.A.J. 2002. On the evolution of cytoplasmic incompatibility in haplodiploid species. Evolution: 1101-1109.

Field A. 2000. MANOVA. In: Wright D.B. (ed.), Discovering statistics using SPSS for Windows. SAGE Publications, London.

Hartl D.L. 1980. Population substructure. In: Principles of Population Genetics. Sinauer Associates, Inc., Sunderland.

Hoffmann A.A. and Turelli M. 1997. Cytoplasmic incompatibility in insects. In: O’Neill S.L., Hoffmann A.A. and Werren J.H. (eds), Influential Passengers. Oxford University Press, Oxford.

Perrot-Minnot M.-J., Cheval B., Migeon A. and Navajas M. 2002. Contrasting the effects of Wolbachiaon cytoplasmic incompatibility and fecundity in the haplodiploid mite Tetranchus urticae. J. Evol. Biol. 15: 808-817.

Reed K.M. and Werren J.H. 1995. Induction of paternal genome loss by the paternal sex-ratio chromosome and cytoplasmic incompatibility bacteria (Wolbachia): a comparative study of early embryonic events. Mol. Reprod. Dev. 40: 408-418.

Saito Y., Sahara K. and Mori K. 2000. Inbreeding depression by recessive deleterious genes affecting female fecundity of a haplo-diploid mite. J. Evol. Biol. 13: 668-678. 
Stouthamer R., Breeuwer J.A.J. and Hurst G.D.D. 1999. Wolbachia pipientis: Microbial Manipulator of Arthropod Reproduction. Annu. Rev. Microbiol. 53: 71-102.

Vala F., Breeuwer J.A.J. and Sabelis M.W. 2000. Wolbachia-induced 'hybrid breakdown' in the twospotted spider mite Tetranychus urticae Koch. Proc. R. Soc. Lond. B 267: 1931-1937.

Vala F., Breeuwer J.A.J. and Sabelis M.W. Sorting out the effects of Wolbachia, genotype and inbreeding on life-history traits of a spider-mite. Submitted to Exp. Appl. Acarol (in press).

Vala F., Weeks A., Claessen D., Breeuwer J.A.J. and Sabelis M.W. 2002. Within- and between-population variation for Wolbachia-induced reproductive incompatibility in a haplodiploid mite. Evolution 56: 1331-1339.

Vala F., Van Opijnen T., Breeuwer J.A.J. and Sabelis M.W. Genetic conflicts over sex ratio: mite-endosymbiont interactions. Am. Nat. (in press).

Van Opijnen T. and Breeuwer J.A.J. 1999. High temperatures eliminate Wolbachia, a cytoplasmic incompatibility inducing endosymbiont, from the two-spotted spider mite. Exp. Appl. Acarol. 23: $871-881$.

Vavre F., Fleury F., Varaldi J., Fouillet P. and Bouletreau M. 2000. Evidence for female mortality in Wolbachia-mediated cytoplasmic incompatibility in haplodiploid insects: epidemiologic and evolutionary consequences. Evolution 54: 191-200.

Werren J.H. 1997. Biology of Wolbachia. Annu. Rev. Entomol. 42: 587-609. 